\title{
Der sprachliche Mischcode im urbanen Milieu und seine Vorläufer
}

Mixed language code in the large city environment and its antecedents

\author{
Anna DASZKIEWICZ ${ }^{1}$ \\ Ateneum-Szkoła Wyższa w Gdańsku
}

\begin{abstract}
Zusammenfassung
Im vorliegenden Beitrag wird dem sprachlichen Mischcode, dem Ethnolekt Rechnung getragen. Nicht nur die sprachlichen Abweichungen des Ethnolekts dem Standarddeutschen gegenüber, sondern auch dessen Vorläufer, historische Bedingungen samt ihren linguistischen Manifestationen werden hier aufgegriffen und näher beleuchtet.

Schlüsselwörter: der sprachliche Mischcode, der Ethnolekt, Abweichungen vom Standarddeutschen, Jugendliche mit türkischem oder arabischem Hintergrund
\end{abstract}

\section{Abstract}

The subject of this paper is a mixed variant of German which arose in large urban agglomerations with participation of mixed youth, indigenous German youth and youth of German lineage. The text outlines historical phenomena, and also linguistic theories which underlie and account for the diversity of mixed code with regard to the standard language.

Keywords: mixed language code, ethnolect, deviations from the standard language, youth with Turkish or Arabic background

1 https://orcid.org/0000-0003-3745-8528

Ateneum-Szkoła Wyższa w Gdańsku, Wydział Neofilologiczny

a.daszkiewicz@ateneum.edu.pl 


\section{Einleitende Bemerkungen}

Die vorliegende Arbeit legt auf eine neue, stark ethnisch geprägte Varietät des Deutschen, den Ethnolekt, Fokus. Es handelt sich hierbei um die Mischvariante des Deutschen und zugleich eine Trendsprache unter Jugendlichen deutscher und nicht deutscher (insbesondere türkischer und arabischer) Herkunft, die in urbanen Ballungsräumen der Bundesrepublik ihren Ausgang nimmt und sprachliche Mischungen aus Elementen der Herkunftssprache ethnolektaler Sprecher und des Deutschen enthält. Da die Betroffenen in der Bundesrepublik aufgewachsen sind, unterscheidet sich ihre Sprechart von der ihrer Vorfahren (der ehemaligen Gastarbeiter). Zudem weicht der ethnisch geprägte Sprechstil von der allgemeinen Jugendsprache $a b$, weil er nicht mehr als eine vorübergehende Sprachmode gilt, seine Aufmerksamkeit erregenden Merkmale an die deutsche Grammatik angepasst werden und die Sprecher selbst einem ähnlichen sozialen Milieu (den sogenannten "Ghettos“) entstammen. Vor diesem Hintergrund wirken die ethnolektalen Sprecher als diejenigen, die zwischen zwei unterschiedlichen Sprachen, Kulturen und Erziehungsstilen hin und hergerissen sind. Deren gemischter Sprechstil ist dementsprechend vornehmlich als Ausdruck der soziokulturellen Identität wahrzunehmen, der Eigeninitiative; ihre Zugehörigkeit weder zur deutschen noch zur türkischen bzw. arabischen Sprache und Kultur manifest zu machen und sich stattdessen sprachlich (und kulturell) im „Zwischenraum“ zu platzieren.

Obwohl im vorliegenden Beitrag die Spezifika des ethnolektalen Sprechstils aufgegriffen und expliziert werden, wird hier nicht ausschließlich das besagte sprachliche Phänomen in seinen Facetten erwogen. Die vorliegende Arbeit hat es nämlich zum Ziel, den Hintergrund sowie einige zur Beschreibung der sprachlichen und kulturellen Hybridität dienende Konzepte aufzuzeigen und zu beleuchten, in denen wie im Fall des Ethnolekts Sprache und Identität in unmittelbarer Beziehung und Wechselwirkung zueinander stehen. Von diesem Blickwinkel her gesehen, verhilft die Darstellung der ausgewählten Konzepte zu Sprachmischungs- und Kreuzungsprozessen dazu, jeweilige Diskurse über sprachliche Standardisierung und NichtStandardisierung theoretisch und sachbezogen $\mathrm{zu}$ untermauern. Dementsprechend wird in der vorliegenden Arbeit zuerst den deutschen und anerkannten linguistischen Arbeiten zum Phänomen Ethnolekt nachgegangen. Ein besonderer Fokus liegt hierbei jeweils auf Strategien zur Bezeichnung der Mischungs- und Kreuzungsprozesse im deutschen Kulturund Kommunikationskontext. Des Weiteren werden themenorientierte 
Ansätze beleuchtet, die anderen Kultur- und Sprachkreisen entstammen und ihren deutschen Pendants als ein wichtiger Bezugspunkt gedient haben. Wie bereits eingangs erwähnt, sollte die vorliegende Arbeit den Diskursen zu sprachlichen Variationsmustern (dem sprachlichen Mischcode) im urbanen Milieu als theoretische Untermauerung dienen sowie über Sprachmischungsund Kreuzungsprozesse Auskunft geben, an denen sich grundsätzlich Jugendliche deutscher und nicht deutscher Herkunft beteiligen und die längst in die Öffentlichkeit eingedrungen und Teil der medialen Realität sind.

\section{Vorläufer der Sprachmischungsprozesse}

Ausgangspunkt für Mischungs- und Kreuzungsprozesse im urbanen Milieu sind Migrationsverfahren und demographische Veränderungen, die die Etablierung von neuen kommunikativen Sozialstilen herbeigeführt haben. Das Erforschen des Sprachverhaltens der Hinzugezogenen sowie dessen Auswirkung auf den Sprachgebrauch der autochthonen Bevölkerung gilt als Domäne der interaktionellen Soziolinguistik oder Kontaktlinguistik. Hierbei liegt der Fokus der Mehrsprachigkeitsforschung grundsätzlich auf einer bestimmten Migrantengruppe: Sowohl in Europa als auch in Nordamerika gewinnen seit Ende der 1970er Jahre die Studien zu Arbeitsmigranten bzw. zu Gastarbeitern die Oberhand (vgl. Nelde, 1998, S. 518). Und dies ist ausgerechnet für die vorliegende Arbeit von hoher Relevanz. Denn es wird hier, wie bereits zuvor angekündigt, dem Sprachgebrauch Jugendlicher mit nicht deutschem Familienhintergrund, also dem der Nachkommen von ehemaligen deutschen Gastarbeitern nachgegangen, die zum großen Teil in der Bundesrepublik aufgewachsen sind. Hierbei weist ihre zur Interaktion mit der Mehrheitsgesellschaft verwendete Kontaktsprache zahlreiche und merkliche Abweichungen vom Standarddeutschen auf. Normferne Codes, die in Bildungskontexten, wo nach wie vor ein "monolingualer Habitus“ (vgl. Gogolin, 1994) herrscht, geringgeschätzt werden, gelten längst auf Spielplätzen und Schulhöfen als „ein selbstverständlicher Teil des unmittelbaren Lebenszusammenhangs, dem sich nur wenige Jugendliche entziehen können“ (Dirim \& Auer, 2003, S. 58). In Anbetracht der zwei sprachlich orientierten Gebote, der Reinigung und Einsprachigkeit, deren Vergegenwärtigung und Anerkennung immer noch in deutschen Schulen gehuldigt wird, gilt Folgendes zu bedenken: „Das Modellieren der Sprache als homogenes Gebilde verstellt den Blick auf die sprachlichen Realitäten 
und die sie konstituierenden heterogenen kulturellen und sprachlichen Ressourcen" (Erfurt, 2003, S. 25).

Die eingängige Annahme, das Migrationsverfahren habe die Grenzverschiebung und/oder Grenzüberschreitung von Sprachen und Sprachvarietäten bewirkt, ist nicht nur auf die heutige Sprachpraxis gemischtethnischer Gruppen zurückzuführen. Für unbestrittene Vorläufer der Sprachmischungsprozesse gelten nämlich Pidgins und Kreolsprachen, die sich aufgrund der Kontakte sowie Konflikte zwischen sprachlich dominanten und dominierten Gruppen herausgebildet haben, woraus auch deren sozialsymbolischer Charakter, sprich deren geringer sozialer Prestigewert hervorgeht. Die vorstehend genannten Kontaktsprachen sind für Kommunikationsanforderungen in den klassischen Kolonialländern charakteristisch. Sie fanden statt, wenn Sprecher anderer Erstsprachen aufeinandertrafen und miteinander agieren mussten. Als typische Kontexte galten hierbei Arbeitssituationen in Handel, Landwirtschaft und Produktion (vgl. Keim, 2012, S. 99). Aufgrund der Unkenntnis der Sprache der Aufnahmegesellschaft wurde ein stark reduziertes und auf den jeweiligen Arbeitskontext bezogenes Sprachkapital entwickelt, dessen Basis Wörter und grammatische Strukturen aus Herkunftssprachen jeweiliger Gesprächspartner stellten. Darauf weist Keim wie folgt hin: „In den klassischen Kolonialländern bildete die Sprache der sozial dominanten Gruppe, der Kolonialmacht, oft die Basis für das Pidgin, zumindest im Bereich des Wortschatzes. Im Bereich der Grammatik wurden die Strukturen aus den Sprachen übernommen, die für die beteiligten Sprecher einfach zu erlernen waren. Wenn z.B. eine Sprache ein komplexes morphologisches System hatte (Genus, Numerus, Kasus, Verbflexion etc.), die andere aber nicht, wurden die komplexen Formen vereinfacht oder weggelassen, z. B. Tempusformen auf Grundformen reduziert und Vor- und Nachzeitigkeit durch Zeitadverbien ausgedrückt" (Keim, 2012, S. 99). ${ }^{2}$

Im Gegensatz zu Pidgin bezeichnet Kreol eine Erscheinung, wenn sich in über längere Zeiträume stabilen Kontaktsituationen pidginisierte Sprachvarietäten in voll ausgebaute Sprachsysteme umwandeln. Als Sonderform von Pidgin wird das Deutsch der ersten Gastarbeiter anerkannt, das dementsprechend als Gastarbeiterdeutsch (zum ersten Mal in einer Pilotstudie von Clyne 1968 beschrieben $^{3}$ ) oder Pidgindeutsch ${ }^{4}$ und im Falle

\footnotetext{
2 Siehe dazu noch (Riehl, 2009, S. 120-121).

${ }^{3}$ Gemeint ist eine Pilotstudie, an der sich insgesamt 15 Ausländer mit spanischer, griechischer, türkischer und slowenischer Erstsprache beteiligt haben. Aufgrund ihrer Aussagen war es für
} 
der ersten Generation türkischer Gastarbeiter als Tarzanca ${ }^{5}$ bezeichnet wird. Im Hinblick auf die Erforschung des Gastarbeiterdeutsch ist eine Reihe von Projekten und Arbeiten entstanden. Erwähnt seien an dieser Stelle etwa das Heidelberger Forschungsprojekt „Pidgin-Deutsch“ (1975) oder die Veröffentlichungen von Bodemann/Ostrow (1975), Meisel (1975), Keim (1978), Yakut (1981), Dittmar (1982), Keim/Nikitopoulos/Repp (1982), Hinnenkamp (1982), Becker/Klein (1984) und Roche (1989). Da das Gastarbeiterdeutsch meist ohne institutionelle Verankerung erworben wurde und demnach stark pidginisierte und fossilisierte Formen des Deutschen aufweist, eignet es sich perfekt zur Markierung des „asozialen Fremden“ und gleichzeitig auch zur Offenbarung der ihn beklemmenden Erfahrungen mit der Diskriminierung, Marginalisierung und Ausgrenzung (Ghettoisierung). Doch die Betroffenen erscheinen als „Akteure mit einer Sprachbiographie und dem kulturellen und ideologischen Gepäck ihrer Migrationsgeschichte auf, als Akteure, die die Sprachpraxis als ein Feld des sozialen Konflikts, der Grenzziehung und Marginalisierung erfahren und deren sprachliches

Clyne möglich, ein Pidginvarietäten-Gefüge festzulegen, das Einwortsätze, Ausfall von Verben, Fehlen des bestimmten und unbestimmten Artikels, Ausfall von Flexionsformen, Verwendung des Infinitivs, Generalisierung des femininen Artikels 'die', Verwendung von 'viel' als Gradpartikel oder 'niks' als Wort- und Satznegation auszeichnen.

4 Siehe hierzu (Riehl, 2009, S. 122-123): „Tatsächlich weist das Deutsch der Gastarbeiter (der 60er und 70er Jahre) Züge eines Pidgins auf. Das gilt vor allem für das Prinzip der Simplifizierung, das ein Grundmerkmal von Pidginsprachen ist. So fallen etwa im Gastarbeiterdeutsch verschiedene syntaktische Kategorien (Artikel, Personalpronomina, Präpositionen, Kopula) weg, Verbformen stehen im Infinitiv, Flexionsendungen fehlen und es besteht eine Tendenz zur Generalisierung des femininen Genus (vgl. Meisel, 1975, S. 21). Das Gastarbeiterdeutsch hat zudem mit Pidgins gemeinsam, dass es nur eingeschränkt gebraucht wird (nämlich am Arbeitsplatz) und auch als Verständigungssprache von Sprechern verschiedener nicht-deutscher Herkunftssprachen untereinander verwendet wird, z. B. von Gastarbeitern aus Italien, Griechenland und der Türkei.“

${ }^{5}$ „Sowohl im Deutschen als auch im Türkischen weichen Muttersprachler in bestimmten Situationen und gegenüber solchen Sprechern, die sich durch die Eigenschaft ausweisen, daß sie der Muttersprache der 'ingroup' gar nicht oder nur beschränkt mächtig sind bzw. deren Gebrauch ihnen aus den unterschiedlichsten Gründen als 'defizitär' unterstellt wird, von den üblichen standardisierten und umgangssprachlich akzeptierten Sprachvarianten der Sprachgemeinschaft ab. Das sprachliche Produkt dieser spezifischen Abweichung können wir als simplifiziert bezeichnen. Unterschiedliche Sprachgemeinschaften haben dafür ihre eigenen Etikette geprägt, wobei ich in Anlehnung an die traditionelle linguistische Bezeichnung, diese Sprechweise als 'Foreigner Talk' bezeichnet habe. Aus Gründen der Unterscheidung habe ich für den türkischen FT den ethnographischen Terminus Tarzanca bzw. Tarzanisch gewählt“ (Hinnenkamp, 1982, S. 154, hervor. im Original). 
Repertoire die kulturellen Formen - und Brüche - ihres grenzüberschreitenden Lebens aufnimmt“ (Erfurt, 2003, S. 25).

\section{Spezifika des sprachlichen Mischcodes im urbanen Milieu}

Es sei vorab angemerkt werden, dass die ersten Gastarbeiter aufgrund einer intensiven Auseinandersetzung mit den Ausgrenzungserfahrungen einen Sprechstil herausgebildet haben, den die bereits zuvor angeführten sprachlichen Besonderheiten kennzeichnen: Ausfall von Artikeln, Präpositionen, Pronomina, Kopulae; Ausfall bzw. Vereinfachung der Verbflexion (Numerus, Tempus, Modus, Person); übermäßige Verwendung des Infinitivs, Verbendstellung, Negationspartikel nix vor dem Verb; Übergeneralisierung mancher semantisch entleerter Verben (sein, haben, machen) oder Ausdruck der Vor- und Nachzeitigkeit durch Temporaladverbien.

Hierbei bilden Spezifika des Gastarbeiterdeutsch den Kern des sprachlichen Repertoires von nachkommenden Migrantengenerationen (vor allem türkischer und arabischer Abstammung, die inzwischen der dritten und vierten Migrantengeneration angehören). Der ethnolektale Sprechstil unmittelbar Betroffener, den türkische Interjektionen und Diskursmarker, Beschimpfungsformeln und Drohrituale, grammatische Reduktionen sowie phonetisch-prosodische Merkmale eines türkisch (ggf. ausländisch) klingenden Akzents ausmachen, ist längst der autochthonen jugendlichen Bevölkerung bekannt und von ihr als Ausdruck einer besonderen Solidarität mit sozial benachteiligten „Ghetto-Kids“ bzw. der von ihnen kreierten Subkultur (als 'Eintrittskarte' zu einer ghettoorientierten Jugendszene), zugleich aber auch - falls ironisch verfremdet und überproportional häufig zitiert - zur Distanzierung von derart "Ghettoisierten“ verwendet. Im engen Zusammenhang damit sei Folgendes angeführt: „Die markierte Verwendung stadtteilsprachlicher Elemente dient vor allem zur sozialsymbolisierenden Darstellung eines groben, aggressiven „Ghetto-Jugendlichen“, von dem sich die jungen Frauen distanzieren, zur Distanzierung von und zum aggressiven Spiel mit deutschen Lehrer/Betreuer und zur Herstellung von IngroupSolidarität gegenüber erwachsenen Deutschen“ (Kallmeyer \& Keim, 2003, S. 44).

\footnotetext{
6 „Das mehrsprachige Phänomen basiert im deutschen Migrationskontext vor allem auf deutschtürkischen Mischungen“ (Canoğlu, 2012, S. 14, hervor. A. D.).
} 
Hierbei werden zur Beschreibung des besagten Sprachphänomens verschiedene Termini und Definitionen gebraucht. Demnach ist der deutschtürkische Mischcode sozial benachteiligter Jugendlicher unter den Namen wie etwa Kanak-Sprak (Zaimoğlu, 1995, 1997), Türkendeutsch (Androutsopoulos, 2001a, S. 1; Kern \& Selting, 2006, S. 239; Kern \& Şimşek, 2006, S. 101-119; Şimşek, 2012, S. 155-180), Türkenslang (Auer, 2003, S. 255), Ghetto/Ausländerdeutsch (Keim, 2004, S. 97; 2008, S. 228), das (hybridolektale) Gemischtsprechen (Hinnenkamp, 2000, S. 96; 2007, S. 195), Kiezdeutsch (Wiese, 2009, S. 782; 2012), Ethnolekt im Gegenwartsdeutsch (LeschSchumacher \& Schumacher, 2009), Turbolekt (Wiese, 2012, S. 17) bekannt und verbreitet. Projekte, die die Mehrsprachigkeitserforschung in migrantisch geprägten Lebenswelten zum Ziel haben, schließen in methodischer Hinsicht an die Ethnographie der Kommunikation im urbanen Milieu, an die interaktionelle Soziolinguistik (Gumperz, 1982, 1994) sowie an die moderne Forschung zum Code-Switching (Rampton, 1995, 1998) an. In Deutschland hat sich vor allem das Konzept des 'sozial kommunikativen Stils' etabliert, das die Mannheimer Forschergruppe um Werner Kallmeyer, Inken Keim und Johannes Schwitalla erarbeitet hat (Kallmeyer (Hrsg.) $1994 \mathrm{u}$. 1995; Schwitalla, 1995; Keim, 1995, 2008 u. 2012). Dem Phänomen von alltagssprachlichen Kreuzungs- und Mischungsprozessen haben sich im Übrigen auch Jannis Androutsopoulos (2001a, 2001b, 2003), Peter Auer (2003), Ĭnci Dirim und Peter Auer (2004) sowie Heike Wiese (2006, 2012) gestellt/verschrieben.

Insbesondere die Konzeption „des kommunikativen sozialen Stils“, die durch die Ethnographie der Kommunikation im Migrantenmilieu, den kultur-soziologischen Ansatz Bourdieus zur stilistischen Differenzierung in der hierarchisch geschichteten Gesellschaft (1979, deutsch 1982) sowie die Theorie der Aushandlung sozialer Ordnung in der Nachfolge des symbolischen Interaktionismus (Strauss, 1968; Schütze, 1987) geprägt ist, erlaubt es, die sozial-symbolische Bedeutung von normfernen Codewerten zu analysieren und zu erhellen. Demnach entscheidet das Gemischtsprechen über soziale Positionierung gemischtethnischer Gruppen und kann als deren wichtiger Identitätsausdruck wahrgenommen werden: „Die Ausprägung von Stil schafft Identitätssymbole, über die auch die Positionierung von Angehörigen eines Milieus bzw. von Akteuren einer sozialen Welt in einem übergreifenden gesellschaftlichen Rahmen möglich wird“ (Kallmeyer \& Keim, 2003, S. 37). Sowohl beim Modell des „kommunikativen sozialen Stils“ als auch bei anderen Entwürfen zur Beschreibung der Mehrsprachigkeit gerät 
die symbolische Bedeutung der Interaktion in den Fokus. Gemeint ist der Input, der die Grenzüberschreitung und die damit einhergehenden Überlappung und Ineinandergreifen von eigenen und fremden Elementen, kurzum das hybride Ganze voraussetzt und determiniert. Dem wird jetzt bei der Charakterisierung von Projekten zur Mehrstimmigkeit des Gesprächs Rechnung getragen.

\section{Ausgewählte sprachliche Manifestationen des Mischcodes Jugendlicher im urbanen Milieu}

\subsection{Language crossing}

In der Sozio- und Kontaktlinguistik werden hybride Praktiken als language crossig ${ }^{7}$ wahrgenommen. Sie treten dann auf, wenn „Sprecherinnen und Sprecher in ihnen 'fremde' Territorien hineinwandern. Im Gegensatz zu klassischen Konstellationen von Zwei- und Mehrsprachigkeit, geht es beim Crossing um unorthodoxe Fälle von Sprachkontakt, um fragmentarische, unnormierte und unerwartbare zwei- oder mehrsprachige Praxis“ (Androutsopoulos, 2003, S. 79, hervor. im Original).

Erste Berichte zum Crossing-Verfahren entstammen englischen Sprachinstituten und sind grundsätzlich mit den Veröffentlichungen von Roger Hewitt (1986) und Ben Rampton $(1995,1998)$ dokumentiert. In den Arbeiten von Hewitt wird zwischen einer ethnozentristischen kulturellen und einer suprakulturellen Modalität unterschieden. Während das erste Konzept eine hybride bzw. synkretistische Kultur bezeichnet, die der Ethnizität als Gesamtprinzip huldigt, aber dennoch auf einheitlichen Wertvorstellungen basiert, wird mit dem zweiten Konzept auf eine außerethnische, von lokalen Besonderheiten emanzipierte Kommunikation verwiesen (vgl. Hewitt, 1998, S. 19). Zur Beschreibung einer komplexen Sprachkontaktkonstellation in einer südenglischen Stadt, wo drei Sprachen, und zwar afro-karibisches Kreol, Panjabi (eine neuindische Sprache) und stilisiertes Assian-Englisch zur Interaktion eingesetzt wurden, griff Ben Rampton auf John. J. Gumperz' Konzept des metaphorischen Code-Switchings zurück. Es sei vorab betont, dass sich Gumperz der allgemeingültigen Auffassung vehement widersetzte, als sei der Sprachwechsel Ausdruck sprachlicher Inkompetenz und zugleich Beweis für jegliche Ausweichmanöver: „Anstatt von einem sprachlichen Defizit auszugehen, wird Code-Switching nach Gumperz als Element sozialer

7 Von Hinnenkamp (1998) wird als deutsches Äquivalent sprachliche Kreuzung bzw. Sprachkreuzung gewählt. 
und rhetorischer Bedeutung verstanden, das von Sprechern gebraucht wird, um ihre Adressaten auf den jeweiligen sozialen und situativen Kontext des Gesprächs aufmerksam zu machen“ (Bücker, 2007, S. 14). Gumperz differenziert zwischen einem situativen und metaphorischen Code-Switching. Bei den besagten Ausprägungen von Code-Switching ist das Element des (Un)erwarteten bzw. die Erwartungshaltung maßgebend. Aufgrund dessen wird der situative Sprachwechsel in multilingualen Kontexten routiniert eingesetzt, als konventionalisiert und denkbar angesehen. Er findet dann statt, wenn eine Situation und herrschende Normen einer Interpretation einer anderen (kulturellen) Perspektive bedürfen, was nicht nur eine Änderung nicht nur bei Gesprächsthemen und -strategien, sondern auch bei Verpflichtungen für die Gesprächsteilnehmer impliziert (vgl. Blom \& Gumperz, 1972, S. 409). Obwohl die betroffenen Interaktanten von einer Sprachperspektive in die andere wechseln, werden jegliche dabei auftretenden Differenzierungen auf lexikalischer und nicht morphologisch-syntaktischer Ebene abgezeichnet (vgl. Blom \& Gumperz, 1972, S. 431). Während das situative Code-Switching situationsbedingte Faktoren wie Gesprächspartner, thema oder -ort voraussetzt, kommt das metaphorische Code-Switching dann vor, wenn eine Stellungnahme zu dem bereits Gesagten abgegeben werden soll, was gewöhnlich auf eine veränderte Absicht, Einstellung oder Gefühlslage beim Sprecher hindeutet (vgl. Bücker, 2007, S. 15). Wie bereits erwähnt, definiert Rampton Language Crossing als Form des von Gumperz beschriebenen metaphorischen Code-Switchings. Dass der Sprachwechsel nicht in Routine-, sondern in Grenzsituationen erfolgt, bringt die besagten Konzepte (das metaphorische Code-Switching und Language Crossing) auf einen gemeinsamen Nenner. Die Tatsache, dass sich am Sprachwechsel Personen aus einer anderen Sprachgemeinschaft beteiligen (im Originaltext ist Crossing "the use of language varieties with social or ethnic groups that the speaker does not normally belong to“ (Rampton, 1995, S. 14) bzw. „the use of a language not normally felt to belong to the speaker" (Rampton, 1998, S. 291)), macht die Überschreitung der ethnisch-sozialen Grenze evident. Dies findet allerdings meist in informellen und darüber hinaus emotional aufgeladenen Situationen/Momenten statt: „[1]Language crossing was located in moments when the ordered flow of social life was loosened and normal social relations could not be taken for granted" (Rampton, 1995, S. 193; siehe hierzu auch Rampton, 1995, S. 281; 1997, S. 65-69; 1998, S. 298-299). Im Unterschied zum routinierten Code-Switching, das die Nebenexistenz von zwei Sprachen im alltäglichen Sprachgebrauch voraussetzt, ist Crossing aufgrund seiner 
Verwendung „nur in begrenzten Momenten außerhalb der dominierenden sozialen Struktur“ nicht besonders „flexibel“ (vgl. Bücker, 2007, S. 15).

\subsection{Transgression}

In Anlehnung an Rampton (1995) und Bakhtin (1971) definieren Ĭnci Dirm und Peter Auer Transgression als Überschreitung einer ethnisch-sozialen Grenze (vgl. Dirim \& Auer, 2004, S. 27). Demnach deutet der Akt einer sprachbezogenen Grenzüberschreitung auf die Usurpierung einer Sprache bzw. einer Sprachvarietät, die den Grenzüberschreitern nicht gehört, hin. So gesehen, gilt Transgression als Act of Identity, die Wahl eines bestimmten Stils aus einem sozialen Stil-Repertoire andersstämmiger Gruppen (vgl. Dirim \& Auer, 2004, S. 39). Hierbei kann das so gesehene Code-Switching (hier: Transgression) eine teilnehmer- oder diskursbezogene Funktion annehmen und heißt demgemäß teilnehmerbezogenes und diskursbezogenes CodeSwitching (vgl. Dirim \& Auer, 2004, S. 158-159).

Es sei an dieser Stelle betont, dass das Konzept von Dirim und Auer ein Sprachverhältnis zwischen türkisch- und deutschstämmigen Jugendlichen in der Bundesrepublik betrifft. Gestützt auf historische Beispiele für Transgressionen dieser Art, also die Übernahme von Versatzstücken sowie ganzen Gesprächsphrasen aus den Sondersprecharten sozial benachteiligter Gruppen wie die Zigeuner (Romani), Landstreicher, fahrenden Händler, Viehhändler (Jenisch, Lekudesch) und Kleinbanditen (Rotwelsch) in die deutsche Studentensprache des 19. Jahrhunderts, kommen Dirim und Auer zur Erkenntnis, dass Transgressionen Distanz von der etablierten Mehrheitsgesellschaft mit ihren standradsprachlichen Normen markieren, die Identifizierung mit den primären Trägern dieser Varietät jedoch grundsätzlich metaphorisch anzusehen ist (vgl. Dirim \& Auer, 2004, S. 27). Denn die beiden Parteien kommen im Alltag nur wenig in Kontakt, nur selten handelt es sich bei Transgressionen um einen assimilatorischen (die gemeinsame Verständigung erleichternden) Spracherwebsprozess. Vielmehr wird mit der Übernahme und Integration von Neuzugängen aus anderen Sprachen und Sprachvarietäten in die eigene Rede die Selbstpositionierung im soziokulturellen Raum, Partizipation an einer großstädtischen Jugendkultur, die im Kontext der Bundesrepublik sprachlich und kulturell türkisch dominiert wirkt, gesichert (vgl. Dirim \& Auer, 2004, S. 30, 146). Kurzum weisen Transgressionen nur bedingt auf die Solidarisierung mit gemischtethnischen und sozial benachteiligten Gruppen hin. Sie scheinen vielmehr auf deren sprachlich-kulturelle Produktivität ausgerichtet zu sein. 
Dass die Identifizierung mit einer bestimmten jugendkulturellen Szene die gleichzeitige Identifizierung mit deren Machern/Schöpfern herbeiführt, ist dabei aber nicht völlig auszuschließen.

So gesehen, sind Transgressionen Beispiele für Sonderformen des Ethnolekts, die dementsprechend als der sekundäre und der tertiäre Ethnolekt eingestuft werden. ${ }^{8}$ Während der sekundäre Ethnolekt die mediale Stilisierung des primären Ethnolekts darstellt, ,in die auch reine ComedyErfindungen wie der Universalartikel dem (dem ist korrekt) und neue Wortbildungen einflossen" (Riehl, 2009, S. 129, hervor. im Original), werden unter dem tertiären Ethnolekt Imitation und Verwendung des sekundären Ethnolekts von jugendlichen Sprechern nicht-türkischer Herkunft verstanden. Die Jugendlichen anderer ethnischer Herkunft (darunter jene mit deutschem Familienhintergrund) greifen auf medial stilisierte Formen des primären Ethnolekts, „um Dinge auszusprechen, die in der eigenen Stimme Gesichtsverletzung oder Tabubruch zum Ausdruck brächten“ (Riehl, 2009, S. 129). Allerdings kann der Türkenslang auch von denjenigen Jugendlichen nicht türkischer Herkunft benutzt werden, die enge Netzwerkbeziehungen zu Trägern des primären Ethnolekts haben. Ihr Gemischtsprechen ist Effekt von sprachlichen Kreuzungen und Mischungen, die tagtäglich in Migrantenvierteln der Bundesrepublik zum Vorschein kommen. Während beim sekundären und tertiären Ethnolekt die Überschreitung der sprachbezogenen Grenze (Transgression) metaphorisch verläuft und wie bereits zuvor angedeutet, nicht unbedingt auf die Affiliation mit den Türken ausgerichtet ist, handelt es sich beim Erwerb der Mischsprache „aus erster Hand“ sowie deren Gebrauch keinerlei um doppeldeutige, sondern ganz umgekehrt - um routinierte, authentische und identitätsstiftende Manifestationen (Rituale).

\section{Abschlussbemerkungen}

In der vorliegenden Arbeit werden wissenschaftliche Arbeiten und Methoden zur Beschreibung des Gemischtsprechens näher gebracht. Hinzu wurden Motivationsgründe aufgezeigt, die gemischtethnische Gruppen zu Kreuzungen und Mischungen von sprachlich codierten Werten aus unterschiedlichen

\footnotetext{
8 Ausgehend von Jannis Androutsopoulos (2001b) entwirft Peter Auer die Typologie der ethnolektalen Formen des Deutschen (2003). In Bezug auf Sprechergruppen und die damit einhergehende Verwendung von ethnolektal geprägten Deutschvarietäten wird nach Auer zwischen dem primären, sekundären und tertiären Ethnolekt unterschieden. S. dazu noch Keim (2011, S. 162).
} 
Kulturkreisen antreiben. Als Verwender der Mischsprache in der Bundesrepublik gelten vorwiegend Jugendliche deutscher und nicht deutscher (vor allem türkischer aber auch arabischer) Abstammung. Von ihnen gebrauchte Deutschvarietäten sind demnach ethnolektal, umgangsund jugendsprachlich geprägt und fungieren als Ausdruck einer besonderen Solidarität mit sozial benachteiligten "Ghetto-Bewohnern“ ggf. der von ihnen gestalteten Jugendszene sowie falls stilisiert (montiert) und ironisch verfremdet völlig Distanzierung davon. Hierbei ist der sozial-symbolische Charakter der Imitation und Verwendung des sprachlich Gemischten für Konzepte zur Beschreibung der Mehrsprachigkeit wie etwa Language Crossing, oder Transgression charakteristisch. Die besagten Konzepte wurden im vorliegenden Beitrag nicht nur in Bezug auf ihre Grundlagen, sondern auch ihre Etablierung in der bundesdeutschen Realität analysiert.

\section{BIBLIOGRAFIE}

Androutsopoulos, J. (2001a). Ultra korregd Alder! Zur medialen Stilisierung und Aneignung von ,Türkendeutsch'. Deutsche Sprache 29, 321-339.

Androutsopoulos, J. (2001b). From the streets to the screens and back again: On the mediated diffusion of ethnolectal patterns in contemporary German. (LAUD, Series A, 522), Essen.

Androutsopoulos J. (2003). jetzt speak something about italiano. Sprachliche Kreuzungen im Alltagsleben. In: J. Erfurt (Hrsg.), „Multisprech“: Hybridität, Variation, Identität (S. 79-109). Oldenburg.

Auer, P. (2003). „Türkenslang“ - ein jugendsprachlicher Ethnolekt des Deutschen und seine Transformationen. In: A. Häcki-Buhofer (Hrsg.), Spracherwerb und Lebensalter (S. 255-264). Tübingen/Basel.

Bakhtin, M. M. (1971). Probleme der Poetik Dostoevskijs. München.

Becker, A. \& Klein, W. (1984). Notes on the internal organisation of a learner variety. In: P. Auer \& A. di Luzio (Hrsg.), Interpretive Soziolonguistics (S. 215-232). Tübingen.

Blom, J.-P. \& Gumperz, J. J. (1972). Social meaning in linguistic structure: code-switching in Norway. In: J. J. Gumperz \& D. Hymes (Hrsg.), Directions in Sociolinguistics. The Ethnography of Communication (S. 407-434). New York.

Bodemann, Y. M. \& Ostrow R. (1975). Lingua franca und Pseudo-Pidgin in der Bundesrepublik: Fremdarbeiter und Einheimische im Sprachzusammenhang. LiLi 5 (17-19/20), 122-146.

Bourdieu, P. (1982). Die feinen Unterschiede. Kritik der gesellschaftlichen Urteilskraft. Frankfurt am Main.

Bücker, T. (2007). Ethnolektale Varietäten des Deutschen im Sprachgebrauch Jugendlicher. (SASI, Heft 9). Münster.

Canoğlu, H. D. (2012). Kanak Sprak versus Kiezdeutsch - Sprachverfall oder sprachlicher Spezialfall? Eine ethnolinguistische Untersuchung. Berlin.

Clarke, J. (1981). Stil. In: J. Clarke \& A. Honneth (Hrsg.), Jugendkultur als Widerstand(2. Aufl., S. 133-157). Frankfurt am Main.

Clyne, M. (1968). Zum Pidgin-Deutsch der Gastarbeiter. Zeitschrift für Mundartforschung, 35, 130-139. 
Dirim, I. \& Auer, P. (2003). „Mit der Zeit versteht man alles“ - Zum ungesteuerten Erwerb des Türkischen durch Jugendliche nicht-türkischer Herkunft. In: J. Erfurt (Hrsg.), „Multisprech“: Hybridität, Variation, Identität (S. 57-78). Oldenburg.

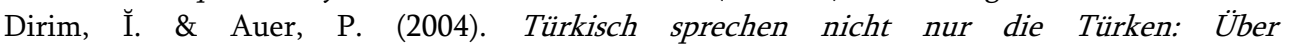
die Unschärfebeziehung zwischen Sprache und Ethnie in Deutschland. Berlin.

Dittmar, N. (1982). „Ich fertig arbeite - nicht mehr spreche Deutsch“. Semantische Eigenschaften pidginisierter Lernervarietäten des Deutschen. LiLi 45, 84-103.

Erfurt, J. (2003). „Multisprech“: Hybridität, Variation, Identität. Oldenburg.

Esteva-Fabregat, C. (1995). Mestizaje in Ibero-America. Tucson, London [span. Original: Mestizaje en Iberoamerica, 1987].

Garciá Canclini, N. (1995). Hybrid Cultures. Strategies of Entering and Leaving Modernity. Minneapolis, London [span. Original: Culturas hibridas: Estrategias para entrar y salir de la modernidad, 1989].

Gogolin, I. (1994). Der monolinguale Habitus der multilingualen Schule. Münster, New York.

Griem, J. (2008). [Art.] Hybridität. In: N. Ansgar (Hrsg.), Metzler Lexikon Literaturund Kulturtheorie (4., aktualisierte und erweiterte Aufl. S. 297). Stuttgart, Weimar.

Gumperz, J. J. (1982). Discourse strategies. Cambridge.

Gumperz, J. J. (1994). Ethnography in urban communication. In: P. Auer \& A. di Luzio (Hrsg.), Interpretive sociolinguistics. Migrants-children-migrant children (S. 1-12). Tübingen.

Heidelberger Forschungsprojekt „Pidgin-Deutsch“ (1975). Sprache und Kommunikation ausländischer Arbeiter. Kronberg, Taunus.

Hewitt, R. (1986). White talk black talk. Inter-racial friendship and communication amongst adolescents. Cambridge.

Hewitt, R. (1998). Ethnizität in der Jugendkultur. In: I. Gogolin et al. (Hrsg.), Pluralität und Bildung (S. 13-24). Opladen.

Hinnenkamp, V. (1982). Foreigner Talk und Tarzanisch. Eine vergleichende Studie über die Sprechweise gegenüber Ausländern am Beispiel des Deutschen und des Türkischen. Hamburg.

Hinnenkamp, V. (1998). Mehrsprachigkeit in Deutschland und deutsche Mehrsprachigkeit. Szenarien einer migrationsbedingten Nischenkultur der Mehrsprachigkeit. In: H. Kämper \& H. Schmidt (Hrsg.), Das 20. Jahrhundert: Sprachgeschichte - Zeitgeschichte (S. 137-162). Berlin.

Hinnenkamp V. (2000). „Gemischt sprechen“ von Migrantenjugendlichen als Ausdruck ihrer Identität. Der Deutschunterricht 5, 96-107.

Hinnenkamp V. (2005). Einleitung. In: V. Hinnenkamp \& K. Meng (Hrsg.), Sprachgrenzen überspringen. Sprachliche Hybridität und polykulturelles Selbstverständnis (S. 7-18). Tübingen.

Hinnenkamp V. (2007). Vom Nutzen einer hybriden Sprache. In: W.-D. Bukow, C. Nikodem, E. Schulze \& E. Yildiz (Hrsg.), Was heißt hier Parallelgesellschaft? Zum Umgang mit Differenzen (S. 175-199). Wiesbaden.

Kallmeyer, W. (1994). Kommunikation in der Stadt. Teil 1: Exemplarische Analysen des Sprachverhaltens in Mannheim (Schriften des Instituts für deutsche Sprache 4.1). Berlin.

Kallmeyer, W. (1995). Kommunikation in der Stadt. Teil 2: Ethnographien von Mannheimer Stadtteilen ( Schriften des Instituts für deutsche Sprache 4.2). Berlin. 
Kallmeyer, W. \& Keim, I. (2003). Eigenschaften von sozialen Stilen der Kommunikation: Am Beispiel einer türkischen Migrantinnengruppe. In: J. Erfurt (Hrsg.), „Multisprech“: Hybridität, Variation, Identität (S. 35-56). Oldenburg.

Keim, I. (1978). Gastarbeiterdeutsch. Untersuchungen zum sprachlichen Verhalten türkischer Gastarbeiter. Tübingen.

Keim, I. (1995). Kommunikation in der Stadt. Teil 3: Kommunikative Stilistik einer sozialen Welt "kleiner Leute» in der Mannheimer Innenstadt (Schriften des Instituts für deutsche Sprache 4.3). Berlin.

Keim, I. (2004). Die Verwendung medialer Stilisierungen von Kanaksprak durch Migrantenjugendliche. Kodikas/Code. Ars: Semiotica, Vol. 26 (2003)/1-2, 97-111.

Keim, I. (2008). Die „türkischen Powergirls“. Lebenswelt und kommunikativer Stil einer Migrantinnengruppe in Mannheim. Tübingen.

Keim, I. (2011). Form und Funktion ethnolektaler Formen: türkischstämmige Jugendliche im Gespräch. In: L. M. Eichinger, A. Plewnia \& M. Steinle (Hrsg.), Sprache und Integration. Über Mehrsprachigkeit und Migration (S. 157-188). Tübingen.

Keim, I. (2012). Mehrsprachige Lebenswelten. Sprechen und Schreiben der türkischstämmigen Kinder und Jugendlichen. Tübingen.

Keim, I., Nikitopoulos, P. \& Repp, M. (1982). Kommunikation ausländischer Arbeiter. Eine Studie zum deutschsprachigen Interaktionsverhalten von griechischen und türkischen Arbeitern. Tübingen.

Kern, F. \& Selting, M. (2006). Einheitenkonstruktion im Türkendeutschen: Grammatische und prosodische Aspekte. Zeitschrift für Sprachwissenschaft 25, 239-272.

Kern, F. \& Şimşek, Y. (2006). Türkendeutsch: Aspekte von Einheitenbildung und Rezeptionsverhalten. In: D. Wolff (Hrsg.), Mehrsprachige Individuen - vielsprachige Gesellschaften (S. 101-119). Frankfurt am Main.

Lesch-Schumacher, B. \& Schumacher M. (2009). „Kiezsprache, Türkenslang, Kanak Sprak? Ethnolekt im Gegenwartsdeutsch«. RAAbits Deutsch/Sprache 61.

Markert, S. (2009). Kommunikative Nähe und Stilisierung von Jugendlichkeit (Arbeitspapiere und Materialien zur deutschen Sprache, Band 36). Mannheim: Institut für Deutsche Sprache.

Meisel, J. (1975). Ausländerdeutsch und Deutsch ausländischer Arbeiter. Zur möglichen Entstehung eines Pidgin. Zeitschrift für Literaturwissenschaft und Linguistik (LiLi), 18, 9-53.

Nelde, P. (1998). Migrantensprachen. In.: G. Holtus, M. Metzeltin \& Ch. Schmitt (Hrsg.), Lexikon der romanistischen Linguistik (Bd. VII, S. 518-526). Tübingen.

Rampton, B. (1995). Crossing. Language and ethnicity among adolescents. London.

Rampton, B. (1997). Sociolinguistics and Cultural Studies: New Ethnicities, Liminality and Interaction. Social Semiotics 1999/9(3), 355-374.

Rampton, B. (1998). Language crossing and the redefinition of reality. In: P. Auer (Hrsg.), CodeSwitching in Conversation (S. 290-320). London.

Riehl, C. M. (2009). Sprachkontaktforschung: Eine Einführung. Tübingen.

Rincón, C. (1994). Die neuen Kulturtheorien. In: B. Scharlau (Hrsg.), Lateinamerika denken. Kulturtheoretische Grenzgänge zwischen Moderne und Postmoderne (S. 1-35). Tübingen.

Roche, J. (1989). Xenolekte: Struktur und Variation im Deutsch gegenüber Ausländern. Berlin.

Schlobinski, P. \& Heins, N.-Ch. (Hrsg.) (1998). Jugendliche und „ihre“ Sprache: Sprachregister, Jugendkulturen und Wertesysteme. Opladen, Wiesbaden. 
Schlobinski, P., Kohl, G. \& Ludewigt, I. (1993). Jugendsprache: Fiktion und Wirklichkeit. Opladen, Wiesbaden.

Schütze, F. (1987). Symbolischer Interaktionismus. In: U. Ammon et al. (Hrsg.), Sociolinguistics. An International Handbook of the Science of Language and Society (3,1. Hbd., S. 520-553), Berlin, New York.

Schwitalla, J. (1988). Die vielen Sprachen der Jugendlichen. In: N. Gutenberg (Hrsg.), Kann man Kommunikation lehren? (Sprache und Sprechen, Bd. 19, S. 167-176), Frankfurt am Main.

Schwitalla, J. (1994). Poetisches in der Alltagskommunikation. In: D. Halwachs et al. (Hrsg.), Sprache, Onomatopöie, Rhetorik, Namen, Idiomatik, Grammatik (S. 227-243). Graz.

Schwitalla, J. (1995). Kommunikation in der Stadt. Teil 4: Kommunikative Stilistik zweier sozialer Welten in Mannheim-Vogelstang (Schriften des Instituts für deutsche Sprache 4.4). Berlin.

Şimşek, Y. (2012). Türkendeutsch: Formen und strukturelle Merkmale des Sprachgebrauchs türkisch-deutscher Jugendlicher aus Berlin. In: B. Jańczak, K. Jungbluth \& H. Weydt (Hrsg.), Mehrsprachigkeit aus deutscher Perspektive (S. 155-180). Tübingen.

Specht, T. (2011). Transkultureller Humor in der türkisch-deutschen Literatur. Würzburg.

Strauss, A. L. (1968). Spiegel und Masken. Die Suche nach Identität. Frankfurt am Main.

Wiese, H. (2006). „Ich mach dich Messer“. Grammatische Produktivität in Kiez-Sprache. http://publikationen.ub.uni-frankfurt.de/frontdoor/index/index/docId/12791 (Zugriff am 12.05.2015).

Wiese, H. (2009). Grammatical innovation in multiethnic urban Europe: New linguistic practises among adolescents. Lingua, 119, 782-806.

Wiese, H. (2012). Kiezdeutsch. Ein neuer Dialekt entsteht. München.

Yakut, A. (1981). Sprache der Familie. Eine Untersuchung des Zweitsprachenerwerbs der türkischen Gastarbeiterfamilien in der BRD. Tübingen.

Zaimoğlu, F. (1995). Kanak Sprak. 24 Mißtöne vom Rande der Gesellschaft. Hamburg.

Zaimoğlu, F. (1997). Abschaum. Die wahre Geschichte von Ertan Ongun. Hamburg.

Zapf, H. (2002). Dekonstruktion des Reinen. Hybridität und ihre Manifestationen im Werk von Ishmael Reed. Würzburg. 
